\title{
Conocimiento, actitud y práctica del médico especialista no ocupacional sobre la valoración de la aptitud médica ocupacional
}

\author{
Knowledge, attitudes and practice of non-occupational physicians on the evaluation of medical occupational \\ aptitude
}

\author{
Miryan Luisa Quintana Maquera' ${ }^{1,2,3, a}$, Juan Carlos Palomino Baldeón 1,4,a, Ray Ticse Aguirre ${ }^{1,2, b}$
}

\section{RESUMEN}

Objetivos: Determinar los conocimientos sobre valoración de aptitud médica para trabajar, la actitud del médico en valorar la aptitud para trabajar y en el seguimiento y control de enfermedades del trabajador, de los médicos especialistas no ocupacionales (MENO). Material y métodos: Estudio observacional, transversal y descriptivo cuyo calculo muestral fue de 163 médicos, se utilizó una encuesta tipo Likert a médicos especialistas clínicos y cirujanos del Hospital Nacional Cayetano Heredia. En las variables cualitativas se determinaron frecuencias, según la aptitud, práctica y conocimiento,y en las variables cuantitativas, medias o medianas según su distribución (normal o asimétrica). Resultados: Se realizó la encuesta a130 MENO, 97 médicos clínicos y 33 cirujanos. El $53,85 \%$ consideró tener un conocimiento sobre valoración de aptitud médica para trabajar, pero solo $16,15 \%$ conoce el concepto de aptitud médica para trabajar. El 24,62\% llegó a reconocer los factores de riesgo en el trabajo. El $16,92 \%$ estimó que las atenciones diarias son de salud ocupacional. Las especialidades que con más frecuencia atienden casos ocupacionales diarios son medicina interna, cardiología, medicina física y traumatología $(\mathrm{p}<0,05)$. Se encontró una buena actitud 93\% $(\mathrm{p}<0,05)$. Conclusiones: No se tiene un conocimiento adecuado, pero si una buena actitud, por lo que se realizaría un trabajo en equipo en la prevención de patologías ocupacionales y no ocupacionales. Es necesario se implemente medicina ocupacional y medio ambiente en la malla curricular del programa de medicina humana de pregrado.

PALABRAS CLAVE: Aptitud, medicina del trabajo, conocimiento, actitud del personal de salud. (Fuente: DeCS BIREME).

\section{SUMMARY}

Objectives: To determine the knowledge on the evaluation of medical aptitude to work, attitude of physicians to evaluate the aptitude to work and the follow-up and control of diseases of workers by non-occupational physicians (NOP). Methods: Cross sectional study that involved 164 physicians. A Likert type survey was applied to clinicians and surgeons at Hospital Nacional Cayetano Heredia. Frequencies were calculated for qualitative and meanmedians were calculated for quantitative variables. Results: The survey was applied to 130 NOP, 97 clinicians and 33 surgeons; $53.85 \%$ had knowledge on how to evaluate medical aptitude to work, but only $16.5 \%$ knew the concept

\footnotetext{
Facultad de Medicina Alberto, Universidad Peruana Cayetano Heredia. Lima, Perú.

Médico especialista de Medicina ocupacional y Medio Ambiente ; ${ }^{\mathrm{B}}$ Médico especialista en Endocrinología y Epidemiólogia
} 
of medical aptitude to work. Risk factors in the work place were known by $24.62 \%$. The $16.92 \%$ of physicians surveyed considered that daily attentions were related to occupational health. The specialties that attended more occupational cases were internal medicine, cardiology, physical therapy and orthopedics $(\mathrm{p}<0.05)$. A good attitude at $93 \%$ was found $(\mathrm{p}<0.05)$. Conclusions: There is no good knowledge but good attitude in evaluating occupational health in this setting. It is recommended to incorporate the topics of occupational health and environmental heath in the medical curriculum in the School of Medicine.

KEYWORDS:Aptitude, occupational medicine, knowledge, attitude of health personnel. (Source: MeSH NLM).

\section{INTRODUCCIÓN}

La valoración de la aptitud para trabajar se define como la evaluación de la capacidad del individuo para realizar su trabajo sin riesgo para su propia salud ni la de otros (1). Esto requiere no sólo la evaluación del estado de salud, sino también un conocimiento profundo de las demandas y requerimientos de las condiciones laborales (2), de los peligros y riesgos encontrados en el puesto de trabajo y su ambiente laboral. En la legislación peruana, la resolución ministerial 312 - 2011, indica que el certificado de aptitud médica ocupacional está determinado por el médico ocupacional, es el resultado de una evaluación médica ocupacional que incluye el estado de salud del trabajador en relación con el puesto de trabajo (3), pero no describe la metodología y actores involucrados para emitir un certificado.

En nuestro país la sociedad de medicina ocupacional y medio ambiente, elaboró una guía de buenas prácticas sobre la valoración de la aptitud medica ocupacional, en la que define cinco criterios para valorar la aptitud medica ocupacional: Clínico ocupacional (donde muchas veces se involucra al médico especialista no ocupacional), conocimiento del puesto de trabajo, en relación con la empresa, éticos y legales (4).

Descartar deficiencias psicofísicas no es función específica del médico especialista ocupacional (MEO), pero decidir si la persona tiene alguna condición que pueda resultar peligrosa en el puesto de trabajo para ella o para terceros sí es específico de la especialidad. Existen factores individuales que aumentan la susceptibilidad para presentar un daño frente a determinados riesgos laborales (5): la edad, trastornos hormonales, el consumo de tabaco, ciertos desórdenes genéticos, trastornos inmunológicos, y enfermedades crónicas no controladas, entre otros. Estos son trastornos abordados por médicos especialistas no ocupacionales, a quienes muchas veces en la consulta, el trabajador les solicita una constancia o certificado de aptitud para continuar trabajando (6).
Un médico sin competencias en medicina ocupacional no tendría la información de las condiciones laborales y experiencia requeridos para valorar la aptitud médica ocupacional. En otros países también se observa un fenómeno similar a lo que ocurre en Perú, por ejemplo, en España muchos médicos asistenciales de atención primaria y médicos especialistas no ocupacionales (MENO) toman decisiones respecto a la valoración de la aptitud laboral de forma deliberada sin información sobre las condiciones laborales del trabajador. Iñaki Korta (7) refiere que "el médico de familia en España, no cuenta con más información que la que les refiere el paciente sobre sus condiciones laborales para sospechar de enfermedad profesional".

Declarar la aptitud médica ocupacional en una reincorporación laboral o el hecho de subsanar una observación de la evaluación médica ocupacional en la emisión de un certificado de aptitud, requiere de un trabajo multidisciplinario entre médicos especialistas no ocupacionales y ocupacionales (8).

El objetivo del trabajo fue determinar los conocimientos sobre valoración de aptitud médica para trabajar, la actitud del médico en valorar la aptitud para trabajar y en el seguimiento y control de enfermedades del trabajador, de los médicos especialistas no ocupacionales (MENO).

\section{MATERIAL Y MÉTODOS}

Estudio observacional, transversal y descriptivo, realizado en médicos especialistas no ocupacionales del Hospital Nacional Cayetano Heredia. Se consideró médico especialista no ocupacional, a los médicos de las áreas clínicas y quirúrgicas que brindan atención a pacientes adultos.

Los criterios de inclusión fueron: médicos que brinden atención a pacientes adultos, y los criterios de exclusión, médicos del área de pediatría y médicos que no llenen correctamente la encuesta. 
Se consideró una población total de 400 médicos, el tamaño de la muestra calculado fue de 163 médicos especialistas no ocupacionales en función de una confianza del $95 \%$ y un margen de error del $5 \%$.

\section{Procedimientos y técnica}

A cada médico especialista no ocupacional se le aplicó un instrumento con 25 preguntas cerradas, y una pregunta abierta, previo consentimiento informado; para las preguntas de actitudes y prácticas se utilizó la escala de Likert. El instrumento está constituido en 4 categorías: el primero son las características de los médicos, el segundo son los conocimientos que tiene sobre aptitud médica para trabajar, el tercero son las prácticas y cuarto las actitudes.

El contenido se validó mediante el método de "Juicio de expertos", utilizando la opinión de 8 especialistas expertos en el tema (05 médicos especialistas ocupacionales, 02 médicos expertos en metodología de investigación y 01 psicólogo).Las sugerencias, hallazgos y recomendaciones dadas por los expertos fueron tomadosen cuenta para realizar los cambios pertinentes del instrumento de investigación. El instrumento se aplicó a una muestra piloto, donde se validó su claridad, lógica y apariencia. La prueba piloto estuvo conformada por 10 médicos residentes de tercer y cuarto año de diferentes especialidades no ocupacionales que prestan atención médica a pacientes adultos.

La encuesta se aplicó en los consultorios médicos de los especialistas, previa aceptación de la entrevista y firma de consentimiento informado.

El trabajo de investigación fue aprobado por el Comité de ética de la Universidad Peruana Cayetano Heredia (UPCH) y aprobado como trabajo de Tesis de postgrado de la Facultad de Medicina de la UPCH.

En las variables cualitativas se determinaron frecuencias, según la aptitud, práctica y conocimiento; en las variables cuantitativas se determinaron medias o medianas según su distribución (normal o asimétrica). Los datos se ingresaron a una base de datos en Microsoft Excel, para una evaluación inicial de consistencia de datos, posteriormente se realizó el análisis de datos en el programa STATA 11.0. Para determinar si las diferencias eran significativas se utilizó el test chi cuadrado para las variables cualitativas, se consideró a $\mathrm{p}<0,05$ como estadísticamente significativo.

\section{RESULTADOS}

Se encuestó a $130(79,75 \%)$ médicos especialistas no ocupacionales de un total de 163 , no se pudo ubicar a 22 médicos, se negaron a llenar la encuesta tres médicos y ocho encuestas estaban incompletas o con errores. El $6,92 \%$ de los médicos refirieron que realizaron algún tipo de formación en Salud Ocupacional como maestría o diplomado. La mayoría de los médicos trabajan en alguna institución como el Hospital de la Solidaridad, o Clínicas afiliadas a una EPS en 77,7\%. Las demás características de los médicos se presentan en la tabla 1.

El $53,85 \%$ consideró tener conocimiento sobre valoración de aptitud médica para trabajar, pero cuando se preguntó sobre la definición de aptitud médica para trabajar respondieron correctamente sólo el 16,15\% (Tabla 2). El 90,77\% de los médicos refirieron que no conocen ni han leído la normativa peruana, la ley 29783, ley de seguridad y salud en el trabajo y Resolución Ministerial 312-2011, que aprueba el documento técnico: protocolo de exámenes médicos

Tabla 1. Características de los médicos del Hospital Nacional Cayetano Heredia.

\begin{tabular}{lcr}
\hline Característica & n & \% \\
\hline Sexo & & \\
$\quad$ Femenino & 40 & 30,77 \\
$\quad$ Masculino & 90 & 69,23 \\
Edad & & \\
$\quad<30$ años & 11 & 8,46 \\
$31-40$ años & 68 & 52,31 \\
41 - 50 años & 38 & 29,23 \\
$>51$ años & 13 & 10,00 \\
Especialidad médica & & \\
Clínicos & 97 & 74,62 \\
Cirujanos & 33 & 25,38 \\
Estudios en Salud Ocupacional & & \\
Maestría & 2 & 1,54 \\
Diplomado & 7 & 5,38 \\
Ninguno & 121 & 93,08 \\
Enseña cursos en Salud Ocupacional & & \\
Si & 8 & 6,15 \\
$\quad$ No & 122 & 93,85 \\
Trabaja en & & \\
H. Solidaridad & 1 & 0,77 \\
Clínica privada & 94 & 72,31 \\
Ambos & 6 & 4,62 \\
Ninguno & 29 & 22,31 \\
\hline & & \\
& &
\end{tabular}


y guías de diagnósticos de los exámenes médicos obligatorios por actividad. El 76,15\% respondió que se debe conocer in situ las tareas o actividades laborales para valorar la aptitud médica. El 24,62\% de médicos llego a reconocer los factores de riesgo en el trabajo, $6,92 \%$ de los médicos llego a reconocer los cinco criterios para valorar la aptitud médica ocupacional establecido por la Sociedad de Medicina Ocupacional y Medio Ambiente de nuestro país.
La percepción de atenciones diarias sobre casos de salud ocupacional fue de $16,92 \%$ de las atenciones. $\mathrm{La}$ frecuencia en que solicitan realizar una constancia de aptitud médica para trabajar es de 6,16\%. El $77,69 \%$ mencionó que pregunta al paciente sobre su ocupación o profesión, El paciente indica que su patología está relacionada al trabajo en $66,92 \%$; solo el $1,54 \%$ de médicos especialistas ocupacionales se llega a comunicar con el médico tratante, para realizar

Tabla 2. Conocimiento sobre concepto de aptitud médica ocupacional.

\begin{tabular}{lcc}
\hline Definición AMO & $\mathrm{n}$ & $\%$ \\
\hline $\begin{array}{l}\text { La evaluación de la capacidad del individuo para realizar su trabajo sin riesgo para su } \\
\text { propia salud o la de otros. }\end{array}$ & 21 & 16,15 \\
$\begin{array}{l}\text { Determinar el ajuste entre la capacidad del trabajador y los requisitos del trabajo. } \\
\begin{array}{l}\text { La evaluación de la capacidad para trabajar eficazmente, evaluar si existe riesgo de } \\
\text { seguridad y salud y valorar la probabilidad de absentismo por enfermedad en el futuro. }\end{array}\end{array}$ & 1 & 0,77 \\
$\begin{array}{l}\text { Proceso bidireccional para identificar la capacidad de trabajo de un individuo (ya sea } \\
\text { física, mental o mixta) y correlacionarla con la naturaleza del trabajo, con re-diseño } \\
\text { del puesto de trabajo si es necesario. }\end{array}$ & 71 & 28,46 \\
TOTAL & 130 & 54,62 \\
\hline
\end{tabular}

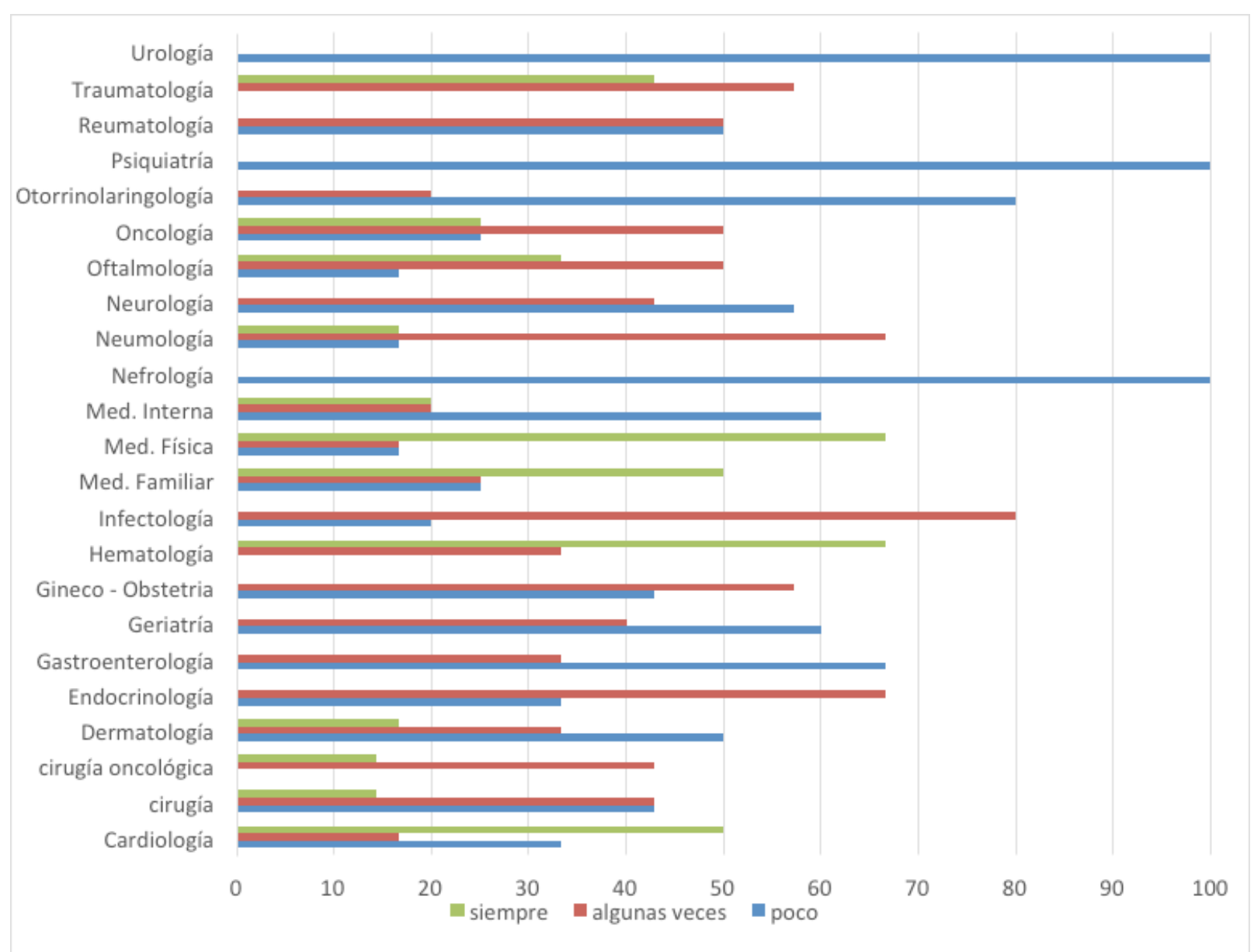

Gráfico 1. Frecuencia diaria de atenciones médico ocupacionales. 
el seguimiento de la patología del trabajador. Las especialidades que perciben tener más atenciones en salud ocupacional son cardiología, medicina interna, traumatología y medicina física $(\mathrm{p}<0,05)($ Gráfico 1$)$.

El 93\% de médicos tuvo una buena actitud sobre la VAMO, $4 \%$ le es indiferente y $3 \%$ tienen una mala actitud. El 90,76\% de médicos estuvo de acuerdo que el certificado de aptitud médica sea realizado por el médico especialista ocupacional. El 76,15\% y 20,77\% estuvieron totalmente de acuerdo y ligeramente de acuerdo respectivamente, en que el médico realice un trabajo en equipo con el médico especialista ocupacional en el seguimiento y control de patologías de sus pacientes, $3.08 \%$ le fue indiferente y $0 \%$ estuvo ligeramente o totalmente en desacuerdo.

El 96,92\% de médicos refirió que estarían de acuerdo en indagar sobre factores de riesgo en el trabajo, cuando el tratamiento convencional no sea efectivo. El 96,92\% estuvo de acuerdo en que debe existir una buena comunicación con el médico especialista ocupacional para determinar una buena aptitud médica. El 81,54\% de médicos consideró que el retorno temprano al trabajo favorece la salud física y mental del paciente, $12,31 \%$ le fue indiferente y $6,15 \%$ no estuvo de acuerdo que sea beneficioso para su paciente. El 96,16\% de médicos estuvieron de acuerdo en que se implemente la medicina ocupacional y medio ambiente en la malla curricular del programa de medicina humana en pregrado.

\section{DISCUSIÓN}

En la percepción de conocimiento sobre valoración de aptitud medico ocupacional (VAMO) alrededor de la mitad de los médicos creen tener un conocimiento adecuado para VAMO, pero de acuerdo a los resultados obtenidos solo el $16,15 \%$ tiene un concepto claro, otros estudios como en Francia, Londres y Amsterdam $(9,10,12,13)$ los MENO y médicos generales, tienen poco conocimiento sobre valoración de aptitud para laborar a pesar de que llevan cursos sobre salud ocupacional, por lo que derivan al trabajador con el médico ocupacional.

En muchas universidades de Europa (9-13) los MENO y médicos generales son capacitados para detectar trabajadores con probable patología ocupacional, así como llenar encuestas sobre factores de riesgo ocupacional, trabajando en colaboración con el médico ocupacional, muchos de estos países en Europa tienen dentro de su programa académico de pregrado cursos sobre medicina ocupacional, por lo que los MENO pueden obtener información ocupacional o detectar en sus evaluaciones medicas probables patologías ocupacionales; cabe resaltar que realizar VAMO corresponde al médico ocupacional, por lo anteriormente mencionado.

En Perú existe un mayor desconocimiento probablemente porque no contamos con un curso de Medicina Ocupacional en la malla curricular del programa de Medicina Humana de pregrado; en nuestro país muchos médicos generales que laboran en SAMO (servicio de apoyo médico ocupacional) realizan evaluaciones médicas (llenar historias ocupacionales) sin tener una base académica mas que la simple inducción o capacitación que recibe de un SAMO, así como muchos MENO llenan historias clínicas sin tomar en cuenta factores de riesgo ocupacional, realizan evaluaciones médicas sin descartar probable patología ocupacional o les llegan referencias médicas (interconsultas) de SAMO para realizar VAMO.

No es acertado que el MENO realice la VAMO; sin embargo, si es necesario que identifique aquellas patologías que probablemente son ocupacionales, por lo que con tratamiento convencional no se curarían o mejorarían (12). Otros estudios como en Bretaña y España $(7,9,13)$ muchos médicos de familia o médicos especialistas no ocupacionales del Instituto Nacional de Salud tienen dificultades para emitir una aptitud médica, ya que no tienen las competencias para realizar la VAMO.

Se encontró una buena actitud de los MENO respecto a la VAMO, en nuestra sociedad la especialidad de Medicina Ocupacional tiene una gran demanda de pacientes de la población económicamente activa, donde existe poco conocimiento sobre su aplicación y trascendencia en el individuo y la sociedad. En Francia la actitud del MENO es positiva en un $82 \%$, pero solo el 36\% entendía bien la labor del MEO (9), es probable que en nuestra sociedad suceda lo mismo los MENO saben que existe la especialidad en medicina ocupacional pero no conocen la labor que realizan.

Se encontró que la mayoría de los MENO encuestados estuvieron de acuerdo en realizar un trabajo en equipo con el MEO para la vigilancia médica de las enfermedades ocupacionales como no ocupacionales del trabajador. En otros estudios, el $71 \%$ de los MENO están dispuestos a trabajar junto con los MEO (14), la vigilancia médica comprende analizar el estado de salud del trabajador así como 
evaluar los riesgos del trabajo, para su prevención de daños a la salud y control de riesgos laborales (3).

Los MENO tiene una actitud positiva en la necesidad de que exista una buena comunicación entre los médicos para determinar una correcta aptitud médica. En Europa (9), la comunicación preferida por los médicos es mediante un documento o carta (92\%) otros medios menos preferidos fue por fax, vía mail, y telefónico. En nuestro país se emiten documentos "interconsultas" la cual en algunas oportunidades los MENO se niegan a llenarla por el mismo desconocimiento así mismo no está referida dentro de una normativa ocupacional.

De los MENO encuestados, menos de la mitad reconoce los factores riesgos que pueden encontrarse en el ambiente laboral, así como la mayoría refiere que se debe conocer los factores riesgo en situ, existe desconocimiento de cuáles son los cinco riesgos laborales básicos como los agentes físicos, químicos, ergonómicos, biológicos y psicosociales entre otros que indican nuestra normativa (3). Según Anna Stern, el 80\% MENO solicitan al MEO información sobre los factores de riesgo laboral en la empresa donde labora sus pacientes y emisión de certificados de aptitud para un retorno o reincorporación laboral, entendemos que los MENO aun recibiendo capacitaciones sobre salud ocupacional, no está dentro de sus competencias reconocer factores de riesgo laborales en el trabajo.

Las especialidades que atienden más frecuentemente casos médico ocupacionales diariamente son medicina interna, cardiología, medicina física y traumatología, estas especialidades son las más frecuentes consultadas por enfermedades crónicas no transmisibles (medicina interna y cardiología) y accidentes laborales (traumatología, medicina física y rehabilitación). En Francia se realizó un estudio con médicos de familia, neumólogos y reumatólogos que son las especialidades más frecuentes que atienden casos ocupacionales, en el estudio (14) recomiendan la necesidad de tener una capacitación inicial y continua de los MENO, así como el uso de herramientas para reconocer enfermedades profesionales para la elaboración de informes médicos, que serían remitidos al MEO o en algún caso médico legal $(17,18)$.

Respecto al retorno temprano al trabajo, la mayoría de MENO está de acuerdo en que el retorno temprano es beneficioso para la salud física y mental del trabajador. En el estudio realizado en el hospital Johns Hopkins y otras sociedades (19) refiere que un retorno temprano al trabajo es beneficioso para la salud y recomiendan un programa que incluya la participación de los proveedores de servicios médicos, profesionales de la seguridad, los trabajadores lesionados y los supervisores del área de trabajo, otros estudios también indican la necesidad de colaboración entre MEO y MENO para realizar un temprano retorno laboral, así como la necesidad de la vigilancia médica del MEO mediante un cronograma de vistas para asegurar la buena salud del trabajador así como su producción para la empresa (20-23).

La mayoría de médicos están de acuerdo en que se implemente en la malla curricular del programa de medicina humana el curso de medicina ocupacional y medio ambiente, ante la necesidad de un conocimiento básico del tema, para la atención oportuna de los pacientes, poder discriminar entre una enfermedad profesional de la no profesional. La necesidad de contar con MEO es escasa ante la gran demanda de la población empresarial, cometiéndose muchas veces errores en emitir una aptitud médica o realizar referencias a otras especialidades para la emisión de aptitud médica.

El instrumento utilizado tiene deficiencias estadísticas, más bien su elaboración fue realizada por un grupo de especialistas con una amplia experiencia en salud ocupacional. Los resultados obtenidos son percepciones que tienen los médicos especialistas no ocupacionales en su práctica diaria.

En conclusión, los MENO no tienen un conocimiento adecuado sobre la VAMO en cambio existe una buena actitud de MENO ante la gran demanda poblacional sobre la valoración aptitud médica y una actitud positiva en trabajar en equipo con el medico ocupacional para la prevención, seguimiento y control de patologías del trabajador.

Se recomienda incluir en las clases de pregrado el curso de medicina ocupacional, se recomienda que las instituciones del estado normen el procedimiento adecuado en la emisión de VAMO en sus diferentes aspectos pre ocupacional, periódico, retiro, temporal y de reincorporación o retorno laboral.

\section{Declaración de financiamiento y de conflictos de intereses:}

El trabajo de investigación fue financiado por el autor principal.Declaramos que ningún autor tiene conflicto de interés con respecto a esta investigación. 


\section{Contribución de autoría:}

MLQM: Diseño del proyecto de investigación, investigación bibliográfica, redacción, análisis, interpretación de resultados y aprobación final JCPB: aporte bibliográfico, análisis y aprobación final. RTA:Metodología, análisis, interpretación de resultados y aprobación final.

\section{Correspondencia:}

Miryan Luisa Quintana Maquera

Calle Tintoretto 125 - San Borja. Lima, Perú.

Correo electrónico: miryan.quintana.m@upch.pe/

miryanluisa_q@hotmail.com.

\section{REFERENCIAS BIBLIOGRÁFICAS}

1. Consol S, Rodríguez MC, Delclos G, Plana M, Benavides F. Criteria and methods used for the assessment of fitness for work: a systematic review. Occup Environ Med. 2007; 64:304-312.

2. Ruiz-Frutos C, García AM, Delclos J, Benavides F. Salud laboral, conceptos y técnicas para la prevención de riesgos laborales. Madrid: Masson; 2007.

3. Ministerio de Salud. Protocolo de exámenes médicos y guía de diagnóstico de los exámenes médicos obligatorios por actividad. Resolución ministerial 312-2011-MINSA. Lima: Dirección General de Salud Ambiental, MINSA; 2011.

4. Consol S, Boix O, Montserrat J, et al. Valoración de la aptitud médica. Madrid: MC Mutual; 2006.

5. Palomino JC, Gomero R, Guía de buenas prácticas para la valoración de la aptitud medica laboral. Lima: SOMOMA; 2014. (Citado el 15 de noviembre). Disponible en: http://somoma.org/ estudio/assets/uploads/files/3da23-Guia--VAMO--SOMOMA-2014-\%284\%29.pdf

6. Magdalena T, Dietl M, Asúnsolo A. Criterios de valoración de la aptitud según profesiones. Med Segur Trab. 2011; 57(223):161-173.

7. Korta I. Reflexiones sobre la incapacidad y aptitud laboral. Sinergias entre medicina evaluadora y medicina laboral. Historia clínica laboral única. La incapacidad laboral, un continuo evolutivo. Med Segur Trab. 2014; 1: 131-141. (Citado el 10 de diciembre del2014) Disponible en:http://gesdoc.isciii. es/gesdoccontroller?action=download\&id=11/09/ 2014-b87a0b34a4

8. Pransky G, Shaw W. Return to Work-From Research to Practice. JOEM. 2007; 49(3):249-251.

9. Verger P, Menard C, Baptiste R, et al. Collaboration between general practitioners and occupational physicians a comparison of the results of two national surveys in France. JOEM. 2014; 56(2): 209- 213.
10. Stern A, Madan I. Optimal communication from occupational physicians to GPs: a cross-sectional survey. Br J Gen Pract. 2012; 62(605):833-839.

11. Gehanno JF, Bulat P. Martinez B, et.al. Undergraduate teaching of occupational medicine in European schools of medicine. Occup. Environ Health. 2014; 87(4):397-401.

12. Buijs P, Amstel R, Dijk F. Dutch occupational physicians and general practitioners wish to improve cooperation. Occup Environ Med. 1999; 56:709-713.

13. Sawney P, Shanellor J. Poor communication between health professionals is a barrier to rehabilitation. Occup Med. 2003; 53(4):246 -248.

14. Arnaud S, Cabut S. ViauA. Souville M, Verger P. Different reporting patterns for occupational diseases among physicians: a study of French general practitioners, pulmonologists and rheumatologists. Occup Environ Health. 2010; 83(3):251-258.

15. Varekamp I, Verbeek JH, van Dijk FJ. How can we help employees with chronic illness to stay at work? A review of interventions aimed at job retention and based on a perspective of empowerment. Int Arch Occup Environ Health. 2006; 80:87-97.

16. Málaga G. Las enfermedades crónicas no transmisibles, un reto por enfrentar. Rev perú med exp salud pública. 2014; 31(1):6-8.

17. Cox RAF, Edwards FC, Palmer K. Aptitud para el trabajo: Los aspectos médicos. Oxford: Oxford Medical Publications; 2000.

18. Thompson J, Brodkin C, Kyes K, et al. Use of a questionnaireto improve occupational and environmental history taking in primary care physicians. JOEM. 2000; 42(12):1188-1194

19. Amato A, Zijlstra F. Toward a climate for work resumption: The nonmedical determinants of return to work. JOEM. 2010; 52(1):67-80.

20. Kushnir T, Luria O. Supervisors' Attitudes toward return to work after myocardial infarction or coronary artery bypass graft. JOEM. 2002; 44(4):331-337.

21. Bernacki E, Guidera J, Schaefer J, et al. A facilitated early return to work program at a large urban medical center. JOEM. 2000; 42(12):1172-1177.

22. Glozier N. Mental ill health and fitness for work. Occup Environ Med. 2002; 59(10):714-20.

23. Anema JR, Jettinghoff K, Houtman I, et al. Medical care of employees longterm sick listed due to mental health problems: a cohort study to describe and compare the care of the occupational physician and the general practitioner. J Occup Rehabil. 2006; 16(1):41-52.

Recibido: $19 / 08 / 2017$

Aceptado: 20/03/2018 